UDC: $371.3:: 811.134 .2$

DOI: https://doi.org/10.18485/beoiber.2017.1.11

\author{
Luiza Valožić1 \\ Doctora por la Universidad de Alicante \\ España
}

\title{
CAMBIO DE CÓDIGO Y USO DE OTRAS LENGUAS POR PARTE DE LOS ALUMNOS EN CLASE DE ESPAÑOL L2
}

\section{Resumen}

En este artículo presentamos los resultados de las observaciones sobre el cambio de código realizado por alumnos en clase de español L2. Partimos de una concepción amplia de la persona bilingüe y multilingüe, definida por la sociolingüística. Las observaciones se llevaron a cabo en un centro de enseñanza de personas adultas de España en el mes de noviembre de 2016.

La utilidad del uso de la L1 y de otras lenguas en clase de L2 o LE es un tema ampliamente debatido entre los investigadores y docentes. Aquí realizamos un breve repaso de las investigaciones que han abordado este tema en los contextos de enseñanza de diferentes lenguas y, sobre todo, del español.

Consideramos que estos usos pueden ser útiles para la enseñanza y el aprendizaje de la lengua meta, sin embrago, también somos conscientes de los potenciales peligros que pueden entrañar si no se desarrollan de manera estratégica. La investigación es, pues, necesaria, para poder definir cuáles son las estrategias adecuadas del uso de L1, y de otras lenguas, en el aula de L2 o LE, que sean beneficiosas para la enseñanza y el aprendizaje.

Palabras clave: enseñanza de español, cambio de código, bilingüismo, multilingüismo.

\section{CODE-SWITCHING AND USE OF OTHER LANGUAGES BY STUDENTS IN THE SPANISH L2 LANGUAGE CLASSROOM}

\begin{abstract}
The paper discusses the results of observations centered on students' code-switching in Spanish classes. Concerning the definition of a bilingual and the multilingual person, we consider it should be based on a broad perspective, defined by sociolinguistics. The observations took place in a public center for adult education in Spain, in November 2016.

The effectiveness of the use of L1 in L2 or FL classes is a subject extensively discussed among researchers and teachers. Here we present some of the work centered on this subject in the context of teaching different languages, Spanish in particular.

\footnotetext{
1 luiza.valozic@gmail.com
} 
We consider that this use can be effective both in teaching and learning the TL, but we are also aware of its potential inconvenience in case it is not developed in a strategic manner. Research is needed in order to define which strategies of use of $\mathrm{L} 1$, and other languages, are appropriate and beneficial for teaching and learning L2 and FL.

Key words: teaching Spanish, code-switching, bilingualism, multilingualism.

\section{Introducción}

La pertinencia o no del uso de L1 en el aula de L2 y LE, y sobre todo la cantidad y la tipología de su uso desde el punto de vista pedagógico, han generado un amplio debate. Desde las últimas décadas del siglo $\mathrm{XX}$, numerosos investigadores y docentes han ido señalando que el planteamiento de uso exclusivo de L2 en el aula de L2 o LE, no parece ser una estrategia siempre apropiada (Macaro 2001, Galindo 2012). Nuestra propia experiencia como aprendices de otras lenguas y como docentes de ELE, nos hace situarnos en la corriente de aquellos que consideran que el uso de L1 (y de otras lenguas) en el aula de L2 o LE, es una estrategia de enseñanza y aprendizaje útil. Por supuesto, aquí cabe matizar, que este uso no debería ser desorganizado, pero sobre todo, no debería representar el último recurso.

A partir de las conclusiones de otros estudios, y de nuestra propia experiencia en el aula de ELE, podemos diferenciar varias tipologías de cambios de código que se dan en el aula de ELE. Por un lado está el cambio de código realizado por el docente, por el otro, el cambio de código realizado por los alumnos en la interacción con el profesor en clase, o con los otros alumnos en una discusión, y en tercer lugar está el cambio de código que realizan los alumnos entre ellos a lo largo de la clase. Estos contextos de cambio de código, se refieren solo a la clase en concreto. Está claro que fuera de la clase, también se da una interacción entre los profesores y alumnos, vinculada a enseñanza, en la que se puede producir el cambio de código. En este sentido, nos referimos, a la comunicación por correo electrónico con el profesor, a la consulta con el profesor después de clase, o en horas de tutoría, o bien, durante las actividades complementarias que se pueden desarrollar fuera del aula, o a través de diferentes canales de comunicación que ofrece internet.

El particular contexto del cambio de código en el que nos centramos aquí, es el cambio de código realizado por los alumnos dentro de la clase de español (L2 $\left.{ }^{2}\right)$, y para tal fin, aplicamos la metodología de la observación participante. Por otra parte, aplicamos la metodología de la encuesta, para conocer qué otras lenguas utilizan los

2 Utilizamos la nomenclatura de español L2, dado que la enseñanza y el aprendizaje de las clases de español que observamos se desarrollan en España. Por lo tanto, nos centramos en diferenciar el contexto de EL2 (aprendizaje en un país hispanohablante), y ELE (aprendizaje fuera de un país hispanohablante). Veremos más adelante, que la mayoría de los alumnos que han participado en estas observaciones, aprenden el español como L3 o L4. 
alumnos en su aprendizaje de ELE. Como veremos más adelante, los alumnos, en cuya actuación se centra nuestro interés, son personas bilingües y multilingües. Las observaciones que presentamos se desarrollaron en el mes de noviembre de 2016 en un Centro Público de Enseñanza de Personas Adultas en Alicante. La magnitud de nuestras observaciones es pequeña, sin embrago, hemos podido ver algunas tendencias de uso de $\mathrm{L} 1$ y de otras lenguas en clase de español.

El estudio del cambio de código en personas bilingües y multilingües se apoya en una larga tradición de investigación desde mediados del siglo XX, iniciada por la sociolingüística, y por obras pioneras como los trabajos de Gumperz (1982) o Poplack (1980) en los que se estudian la tipología de los cambios de código y los contextos en los que se desarrollan.

Los datos de la investigación han demostrado que el cambio de código es un comportamiento natural en personas bilingües y multilingües.

\section{Investigación sobre la presencia de otras lenguas en el aula de L2/LE}

La investigación de la adquisición de segundas lenguas (en adelante ASL) ha aportado numerosos datos sobre el complejo proceso de la enseñanza y el aprendizaje de segundas, y otras lenguas. Además de esta disciplina, se han ocupado de su estudio también la psicolingüística y la sociolingüística. En las últimas décadas, la ASL y la sociolingüística han experimentado diferentes puntos de acercamiento. En el trabajo de Moreno (1994), encontramos una amplia revisión de los aportes que la sociolingüística ha hecho a la enseñanza de lenguas, e indirectamente a otras disciplinas lingüísticas. Algunos de ellos son, por ejemplo, la variación de la lengua, los cambios lingüísticos y la influencia de unas lenguas sobre otras (1994: 110). Por otra parte, en este trabajo el autor cita las observaciones de S. Pit Corder de 1973, en las que destaca que se obtienen los mejores resultados en la enseñanza, cuando el contenido lingüístico de los cursos se acerca a las necesidades funcionales de los estudiantes, lo cual apunta a la necesidad de estudiar la lengua en su contexto social (Moreno 1994: 111). Dentro del contexto de la sociolingüística, también hace referencia a la aportación de concepciones teóricas de la etnografía de la comunicación, como son: comunidad de habla, competencia comunicativa, repertorio, situación comunicativa, acontecimientos y actos comunicativos y rutina (Moreno 1994: 114).

Dentro del contexto de la enseñanza y aprendizaje de L2 y LE, también se ha estudiado el tema del cambio de código dentro del aula, como veremos con más detalle a continuación. En la mayoría de los casos, estos estudios se han centrado en el estudio del uso de la L1 en clase de inglés como L2. Sin embrago, en los últimos años se han realizado estudios de la presencia de L1 en el aula de L2 de otras lenguas. La bibliografía sobre este fenómeno es vasta, por lo que expondremos a continuación solo unas breves 
líneas sobre algunos estudios que abordan la presencia de otras lenguas en el aula de L2 o LE.

En la línea de investigadores que consideran beneficioso el uso de L1 en el aula de L2, podemos citar a Macaro (2001: 532), quien sostiene que L1 no debería ser desterrada del aula de L2, y que, además, el no recurrir a su uso, puede privar a los alumnos de una estrategia de aprendizaje útil.

En el trabajo de Butzkamm (2007: 71-72), también encontramos una visión positiva del uso de L1 en el aula de L2. El autor sostiene que el conocimiento de la L1 y de otras lenguas, representan habilidades y competencias que son útiles para la generación de nuevos conocimientos. El uso de la L1 en el aprendizaje es una tendencia natural, por lo tanto, debería ser aprovechada para la adquisición de nuevos conocimientos (Butzkamm 2007: 84).

Jessner (2008) expone los detalles de un nuevo planteamiento teórico, la adquisición de terceras lenguas (ATL), presentado por Herdina y Jessner en 2002. En el mencionado trabajo, las autoras presentan el 'modelo dinámico de multilingüismo', de acuerdo con el que un sistema multilingüe no es lineal, cambia con el tiempo, y es reversible (Jessner 2008: 25).

La autora cita algunos estudios que han demostrado la evidencia del uso por parte de los aprendices de L3, no solo de L1, sino también de L2. Estos trabajos se han centrado en el estudio del aprendizaje de lenguas indoeuropeas. Los aprendices cuyas L1 no son de una tipología similar con la L2 o la L3, tienden a transferir sus conocimientos de su L2, o en el caso de los bilingües, de la L1, de tipología similar a la L2 (Jessner 2008: 31).

Dentro del contexto de la enseñanza de ELE, también se han desarrollado estudios, que han prestado especial atención a la presencia de L1 y otras lenguas en el aula de ELE. El estudio de Galindo (2012) abarca una investigación amplia del uso de L1 en el aula de español como L2 y LE, que se centra tanto en estos usos por parte de los profesores como de los alumnos. Además, en este trabajo se presenta una revisión detallada de la bibliografía que se ocupa del estudio de la presencia de L1 en la enseñanza de otras lenguas, y de manera destacada, del inglés.

Otros estudios que también abordan el tema del uso de L1 y otras lenguas en el aula de ELE, son el estudio de Palikuća y Novelliere (2012), que se centra en el análisis del uso del inglés por parte de profesores de ELE, Jiménez (2012), que adopta el marco teórico del análisis conversacional, y se centra en el estudio de la 'alternancia códica' de L1 por parte del profesor en clase de ELE, o el estudio de Gutiérrez (2013), enfocado al análisis de las opiniones de los profesores de ELE sobre el uso de otras lenguas en el aula para la enseñanza.

\section{Marco teórico y metodológico}

Las observaciones que presentamos aquí se fundamentan en las bases teóricas de la sociolingüística. 
En primer lugar, cabe destacar la descripción de los elementos que intervienen en la enseñanza y el aprendizaje de lenguas. En este sentido, seguimos la delimitación establecida por Moreno Fernández (2000). El autor diferencia tres elementos, a los que denomina respectivamente como el objeto del aprendizaje lingüístico, esto es la lengua meta, el sujeto del aprendizaje lingüístico, el estudiante o aprendiz, y el tercer elemento es el contexto social del aprendizaje (2000: 7-9). De acuerdo con esta descripción, nos centraremos aquí en el sujeto del aprendizaje lingüístico, esto es en el alumno, y, específicamente, en el uso que hacen los alumnos del cambio de código en clase de español, y en el uso de otras lenguas, durante su aprendizaje de español L2.

Las definiciones del bilingüismo, y del multilingüismo, no están exentas de polémica. Nos basamos en una concepción amplia del bilingüismo, según la cual, para ser considerado bilingüe, no es indispensable el uso alternativo de lenguas, sino que es suficiente que estas lenguas sean conocidas por el hablante. El individuo bilingüe, además, forma parte de una comunidad de habla y es condicionado por factores socioculturales de esa comunidad (Gimeno y Gimeno 2003: 25).

Entendemos por cambio de código el uso de dos lenguas por un hablante durante el transcurso de una conversación, que puede producirse en forma de elementos léxicos, proposiciones u oraciones. El cambio de código no presenta ningún tipo de integración respecto a la lengua base, fonológica, morfológica, ni sintáctica. Además, el cambio de código siempre implica algún grado de competencia bilingüe (Gimeno y Gimeno 2003: 109-115).

En cuanto a la metodología empleada en nuestras observaciones, se trata de la observación participante. Por lo tanto, adoptamos el rol de profesor en un aula de español, y durante la impartición de clase, observamos el comportamiento comunicativo de nuestros alumnos, y nos centramos en la tipología de cambios de código que realizan en clase.

Aparte de la observación participante, también realizamos una encuesta a los alumnos, en la que les planteamos preguntas sobre sus conocimientos lingüísticos y sobre el uso de otras lenguas, durante su aprendizaje del español como L2.

Cabe señalar que se trata de unas observaciones que nos han aportado principalmente datos cualitativos. La dimensión de las observaciones es muy pequeña, sin embargo, sí que hemos podido observar diferentes tipos de cambios de código en clase de EL2.

\section{Observaciones en clase}

Como hemos dicho al principio, nos centramos en este trabajo en el cambio de código que realizan los alumnos en el aula de EL2. Por otro lado, también nos centramos en observar el uso de otras lenguas conocidas por el alumno en su aprendizaje de español, que comentaremos en el apartado siguiente. 
Hemos destacado que nuestras observaciones se basan en la observación participante. Adoptamos el papel de profesor, y durante la impartición de clase, observamos los cambios de código que se daban, y de qué tipo eran.

El contexto de enseñanza de nuestras observaciones son las clases de EL2 impartidas en una escuela pública de educación de personas adultas en Alicante. Los alumnos proceden de diferentes partes del mundo, y son personas que inmigraron a España. Es importante destacar que, en términos de conocimientos lingüísticos, la mayoría de los alumnos son personas bilingües, que aprenden español como L3. El número de alumnos por clase era de alrededor de veinte personas. Llevamos a cabo nuestras observaciones en el mes de noviembre de 2016.

Las observaciones las realizamos durante dos clases de dos horas. Una de las clases correspondía al nivel de español A1, y la otra al nivel de español B1. En cuanto al contenido de las clases que impartimos, seguimos con el programa regular, a partir de la previa consulta con la profesora que se encarga de impartir estas clases. La lengua vehicular de clase era el español. No quisimos cambiar el contenido de las clases, ni acudir a la posibilidad del uso de otras lenguas por nuestra parte, con el objetivo de poder observar un contexto lo más aproximado posible a la realidad diaria del aula.

Más adelante veremos que la mayoría de los alumnos tiene el francés como L2. Aunque por nuestra parte, poseemos ciertos conocimientos del francés, en ningún momento acudimos a esta posibilidad, de nuevo, con el objetivo de observar la interacción comunicativa espontánea.

A continuación exponemos las observaciones de las clases de español A1 y B1. En los dos contextos, observamos los mismos usos de L1 (árabe), y los mismos cambios de código, a excepción de uno observado en clase de B1, que comentaremos más tarde con mayor detalle. Además del cambio de código, también constatamos, como es natural, la interacción espontánea entre alumnos en su L1. Este tipo de interacciones se da tanto en clase de español A1 como en clase de español B1. En cuanto a la interacción entre los alumnos en su L1, se usa de los siguientes modos: por parte de los alumnos con mayores conocimientos de español para las explicaciones a sus compañeros, para la realización de preguntas a los alumnos que poseen mayor conocimiento de español por parte de otros alumnos, durante la realización de las tareas de clase y como vehículo espontáneo de comunicación entre los alumnos.

Cabe mencionar aquí los contextos de uso de $\mathrm{L} 1$ recogidos en la investigación de Galindo (2012), por parte de alumnos de español, que son los siguientes: para expresar opiniones, conseguir un clima de entendimiento y para la construcción de la intersubjetividad, que permite a los alumnos realizar las tareas y crear estructura entre ellos (2012: 163).

El primer tipo de cambio de código que observamos, es el cambio de código en L1 por parte de los alumnos que primero hacen preguntas al profesor en español, y luego se encargan de realizar las explicaciones a sus compañeros que no entienden algo comentado en clase. Está relacionado con este cambio de código, el cambio de código de 
L1, de tipo léxico, realizado por los alumnos con mayores conocimientos, que usan la L1 para decir la traducción de una palabra nueva en español al resto de sus compañeros.

También anotamos un cambio de código en L2. Se trata del cambio de código que observamos solo en la clase de B1, y al que hemos aludido anteriormente. El cambio de código se produce cuando una alumna reconoce una estructura gramatical del español, que le parece similar a una estructura gramatical en francés, y se dirige en francés, su L2, a sus compañeros, para comentarles esta similitud.

Más tarde, la alumna nos hace su consulta sobre lo que le ha parecido una similitud gramatical entre el español y el francés. La duda se centraba en el hecho de que la contracción preposicional del español, le recuerda a la regla de liaison en francés, relacionada con la pronunciación de palabras que terminan en consonante y empiezan por una vocal. Por lo tanto, equipara el caso del español con la regla del francés y lo relaciona con la presencia de dos vocales seguidas. Pero, observa en otro caso que la regla no se cumple. Acto seguido aclaramos la duda de la alumna, y subrayamos que la regla en el español no tiene que ver estrictamente con la acumulación de vocales sino con las preposiciones y artículos. Este caso nos parece particularmente interesante, ya que coincide con el hecho al que han aludido otros investigadores (Jessner 2008), y que consiste en transferir los conocimientos de L2 a la hora de aprender una L3, en este caso, el español.

\section{Datos de la encuesta}

Con el objetivo de recoger datos sobre el uso de otras lenguas por parte de los alumnos, llevamos a cabo una encuesta de ocho preguntas de tipo abierto, donde solo una de las preguntas contenía opciones de respuesta de tipo sí o no. La misma encuesta la elaboramos en español, francés e inglés, con el fin de facilitar su cumplimentación a los alumnos que no poseen todavía el suficiente conocimiento de español. Comentaremos aquí solamente algunos de los datos recogidos, debido, por una parte, a que en algunos casos no obtuvimos respuesta, y por otra, a que no es nuestro objetivo en este trabajo tratar con detalle los datos obtenidos, ya que las dimensiones del estudio son muy reducidas.

En la clase de español A1 recogimos datos de catorce alumnos, y en la clase de B1, de cuatro. La edad de los alumnos es entre los 18 y los 50 años. En la clase de A1 recogimos las respuestas de siete alumnas y de siete alumnos. En la clase de B1, recogimos las repuestas de tres alumnas y un alumno.

Nos parece de particular interés el hecho de que los alumnos presentan un perfil mayoritario de hablante multilingüe. La mayoría aprende el español como L3, o incluso L4, como podemos ver en el siguiente cuadro 1.1. 
Cuadro 1.1. Perfil lingüístico de los alumnos de A1

\begin{tabular}{|c|c|c||}
\hline PERFIL LINGÜÍSTICO & ‥ ALUMNOS & $\%$ \\
\hline \hline $\begin{array}{c}\text { L1 ÁRABE } \\
\text { L2 FRANCÉS } \\
\text { L3 ESPAÑNL }\end{array}$ & 8 & 57,14 \\
\hline \hline $\begin{array}{c}\text { L1 ÁRABE } \\
\text { L2 FRANCÉS } \\
\text { L3 INGLÉS } \\
\text { L4 ESPAÑNOL }\end{array}$ & 4 & 28,58 \\
\hline \hline $\begin{array}{c}\text { L1 WÓLOF } \\
\text { L2 FRANCÉS } \\
\text { L3 ESPAÑNL }\end{array}$ & 1 & 7,14 \\
\hline \hline $\begin{array}{c}\text { L1 GEORGIANO } \\
\text { L2 ESPANNOL }\end{array}$ & 1 & 7,14 \\
\hline \hline TOTAL & 14 & 100 \\
\hline
\end{tabular}

Si sumamos los porcentajes del cuadro 1.1 de los alumnos que estudian el español como L3, obtenemos un porcentaje de $64,28 \%$. El porcentaje de $28,58 \%$ corresponde a los alumnos que estudian el español como L4, y un 7,14\% a los alumnos que estudian el español como L2.

Una de las respuestas que más nos interesaba de la encuesta, es qué tipo de diccionario usan los alumnos en su aprendizaje de español. Los datos de las respuestas de los alumnos, los podemos ver en el cuadro 1.2. Aunque en el cuadro consta solo una dirección del diccionario, por ejemplo, español-L1, se sobreentiende que los alumnos utilizan diccionarios bidireccionales.

En el cuadro 1.2 vemos que la mayoría de los alumnos, un 42,86\%, utiliza un diccionario de su L1 y el español. Nos ha parecido interesante que cuatro alumnos hayan indicado en el espacio donde se les preguntaba por el uso del diccionario, la ayuda que les proporcionan sus amigos y familiares. En el caso de los familiares, los alumnos indicaron la ayuda de miembros más jóvenes, lo cual es de esperar, dada su integración lingüistica y social a través de la escolarización obligatoria.

Cuadro 1.2. Tipo de diccionario usado por los alumnos de A1

\begin{tabular}{|l||c|c|}
\hline TIPO DE DICCIONARIO & N.․ ALUMNOS & $\%$ \\
\hline
\end{tabular}




\begin{tabular}{|l|c|c|}
\hline ESPAÑOL-L1 & 6 & 42,86 \\
\hline \hline ESPAÑOL-L2 & 1 & 7,14 \\
\hline \hline $\begin{array}{l}\text { ESPAÑOL-L1 y } \\
\text { ESPAÑOL-L2 }\end{array}$ & 1 & 7,14 \\
\hline \hline $\begin{array}{l}\text { ESPAÑOL-L2 } \\
\text { ESPAÑOL MONOLINGÜE }\end{array}$ & 1 & 7,14 \\
\hline \hline $\begin{array}{l}\text { ESPAÑOL-L1 } \\
\text { ESPAÑOL L2 } \\
\text { L1-L3 (INGLÉS) }\end{array}$ & 1 & 7,14 \\
\hline \hline $\begin{array}{l}\text { ESPAÑOL-L2 } \\
\text { AMIGOS Y FAMILIARES }\end{array}$ & 1 & 7,14 \\
\hline \hline $\begin{array}{l}\text { ESPAÑOL-L1 } \\
\text { AMICOS y FAMILIARES }\end{array}$ & 2 & 14,30 \\
\hline \hline $\begin{array}{l}\text { ESPAÑOL-L1 } \\
\text { ESPAÑOL-L2 } \\
\text { AMIGOS y FAMILIARES }\end{array}$ & 1 & 7,14 \\
\hline \hline TOTAL & 14 & 100 \\
\hline
\end{tabular}

Pasamos ahora a comentar los datos a partir de la encuesta a alumnos de la clase de español de nivel B1. Primero, recogemos en el cuadro 1.3 los datos del perfil lingüístico de los alumnos. De nuevo observamos, que el perfil lingüístico de los alumnos es predominantemente multilingüe, ya que de los cuatro alumnos entrevistados, solo uno estudia el español como L2.

Cuadro 1.3. Perfil lingüístico de los alumnos de B1

\begin{tabular}{|c|c|c||}
\hline PERFIL LINGÜÍSTICO & N.․ ALUMNOS & $\%$ \\
\hline \hline $\begin{array}{c}\text { L1 ÁRABE } \\
\text { L2 FRANCÉS } \\
\text { L3 ESPAÑOL }\end{array}$ & 2 & 50 \\
\hline \hline $\begin{array}{c}\text { L1 UCRANIANO } \\
\text { L2 RUSO }\end{array}$ & 1 & 25 \\
\hline
\end{tabular}




\begin{tabular}{|c||c||c|}
\hline $\begin{array}{c}\text { L3 INGLÉS } \\
\text { L4 ESPAÑOL }\end{array}$ & & \\
\hline \hline $\begin{array}{c}\text { L1 RUMANO } \\
\text { L2 ESPAÑOL }\end{array}$ & 1 & 25 \\
\hline \hline TOTAL & 4 & 100 \\
\hline
\end{tabular}

Los datos referentes al uso de los diccionarios por parte de los alumnos de B1, se presentan en el siguiente cuadro 1.4 .

Cuadro 1.4. Tipo de diccionario usado por los alumnos de B1

\begin{tabular}{|l||c||c|}
\hline TIPO DE DICCIONARIO & N.. ALUMNOS & $\%$ \\
\hline \hline ESPAÑOL MONOLINGÜE & 3 & 75 \\
\hline \hline GOOGLE TRANSLATE & 1 & 25 \\
\hline \hline TOTAL & 4 & 100 \\
\hline
\end{tabular}

El cuadro 1.4 nos indica una superioridad significativa del uso de un diccionario monolingüe de español en este nivel de aprendizaje. El alumno que señaló que utiliza la herramienta de Google Translate, nos comentó que la utiliza principalmente para las traducciones de su L1 y el español.

Aunque, como ya hemos subrayado, los datos de nuestras observaciones son de un tamaño muy pequeño, la comparación de este elemento observado, el tipo de diccionario utilizado por los alumnos del nivel de $A 1$ y $B 1$, nos indican una diferencia importante. Mientras que los alumnos del nivel de A1 utilizan mayoritariamente diccionarios de español y su L1 (42,86\%), los alumnos del nivel B1, muestran una preferencia por los diccionarios monolingües en español (75\%).

Este dato coincide con los hallazgos de otros investigadores, que apuntan que los alumnos, según adquieren mayor competencia en la lengua meta, muestran menor uso, y de alguna manera, menor dependencia del uso de su L1 en el aprendizaje de la lengua meta (Galindo 2012: 142-145).

Con el objetivo de recoger más información sobre el uso que hacen los alumnos de otras lenguas en el aprendizaje de español, pero sobre todo, de conocer sus reflexiones en cuanto a este tema, incluimos en el cuestionario la siguiente pregunta: "Usas el conocimiento de otras lenguas a la hora de aprender español. ¿Podrías poner un ejemplo?". Solamente obtuvimos cinco respuestas (del total de catorce) a esta pregunta 
por parte de los alumnos del nivel de A1. En el nivel B1, no obtuvimos respuestas a esta pregunta. En las cinco respuestas, los alumnos de L1 (árabe), indicaron que para este fin utilizan su L2 (francés), y el ejemplo era el uso del diccionario de L2-español.

En relación con este hecho, encontramos un planteamiento de reflexión similar a los alumnos en el trabajo de Galindo (2012). En dicha investigación, una de las preguntas que se planteó a estudiantes de español como L2 fue la siguiente: “¿Qué papel tiene la L1 en el aprendizaje de una L2?». Según los datos de la encuesta mencionada, las respuestas de los alumnos se distribuyen de la siguiente manera: ayuda a aprender más rápido $(43,8 \%)$, es fuente de transferencias positivas y negativas (20,7\%), ninguno (16\%), ralentiza el aprendizaje $(7,1 \%)$, no sé $(6,5 \%)$, no entiendo la pregunta $(5,9 \%)$. La autora asimismo destaca que esta fue una de las preguntas que más dificultades de respuesta planteó a los alumnos (Galindo 2012: 143-144).

Por otra parte, en esta misma investigación expone las reflexiones de algunos alumnos, cuyas L1 no comparten rasgos con el español, y que decían que recurren a otras lenguas que conocen en su aprendizaje del español. También incluye la aportación de una profesora que destaca el valor positivo de esta 'multicompetencia' de los alumnos (Galindo 2012: 145).

Subrayamos este hecho, porque nos parece relevante, por un lado, conocer las reflexiones de los alumnos sobre su proceso de aprendizaje del español, y otras lenguas, y por el otro, conocer hasta qué punto el uso de la L1 y de otras lenguas es una herramienta que los alumnos utilizan de manera consciente.

\section{Conclusiones}

La observación de los usos de diferentes lenguas en el aula de español L2 por parte de alumnos bilingües y monolingües, nos ha proporcionado evidencias sobre las diferentes estrategias de comunicación que utilizan. Dentro del contexto del aula de español como L2 en España, hemos podido observar los siguientes usos de la L1 por parte de los alumnos, tanto en clase del nivel A1 como en el nivel B1: uso de L1 por parte de los alumnos con mayor competencia en español para explicar puntos tratados en clase a otros compañeros, preguntas a los alumnos con mayor competencia por parte de los alumnos con menor competencia en español, en la realización de las tareas de clase y como vehículo de la comunicación espontánea.

Por otra parte, el cambio de código de L1 en el aula se da por parte de los alumnos que primero hacen preguntas al profesor en español, y explican a sus compañeros aquello que no habían entendido. También se da un cambio de código léxico de L1, realizado por los alumnos con mayores conocimientos, que usan la L1 para decir la traducción de una palabra nueva a sus compañeros. 
Registramos también un cambio de código en $\mathrm{L} 2$ en la clase de $\mathrm{B} 1$ que se produce cuando una alumna comenta en francés, su L2, a sus compañeros, una característica gramatical del español que le parece similar a otra característica del francés.

Otro hecho que hemos podido constatar, es la transferencia positiva de conocimientos de L2 a la L3, también descrita por otros investigadores.

Los datos de la encuesta nos proporcionan la evidencia de que, en este contexto de aprendizaje, los alumnos son mayoritariamente bilingües y multilingües, y aprenden el español como L3 o L4. El análisis de los datos sobre el uso de los diccionarios de los alumnos, nos indica que en el nivel más básico L1, un 42,86\% utiliza un diccionario español-L1, mientras que en el nivel B1, el $75 \%$ de los alumnos que cumplimentaron la encuesta utiliza un diccionario de español monolingüe. Esta tendencia, coincide con la señalada por otros autores, de que los alumnos presentan menor dependencia, o uso de su L1, según va aumentando su competencia en la lengua meta.

A partir de nuestra propia experiencia como aprendices de otras lenguas y como docentes de ELE, hemos podido ver que el uso de L1 y de otras lenguas en el aula de L2 o LE puede ser una estrategia de enseñanza y aprendizaje útil. Sin embargo, no debemos desestimar las potenciales desventajas de esta estrategia, sobre todo si su uso carece de cualquier tipo de organización, y si se recurre a ella como último recurso, ya que entonces el desarrollo de la clase y el propio proceso de aprendizaje se pueden ver afectados de manera negativa. Por ello, hemos de profundizar en la investigación de los diferentes contextos y usos, con el propósito de deslindar qué estrategias de uso de L1 y de otras lenguas en el aula de L2 y LE pueden ser beneficiosas para el proceso de aprendizaje de la lengua meta.

\section{Agradecimientos}

Deseo expresar mi inmensa gratitud al equipo directivo y docente del Centro Público de Educación de Personas Adultas 'Profesor Alberto Barrios' por haberme prestado su colaboración y ayuda, para la realización de las observaciones aquí presentadas.

También agradezco enormemente la colaboración y la participación de los alumnos con los que he tenido el placer de trabajar.

\section{BIBLIOGRAFÍA}

Butzkamm, Wolfgang. "Native Language Skills as a Foundation for Foreign Language Learning". Wolf Kindermann (ed.), Transcending boundaries. Essays in honour of Gisela Hermann Brennecke. Berlin: Lit Verlag, 2007: 71-85. Print. 
Galindo Merino, M. a Mar. La lengua materna en el aula de ELE. Málaga: ASELE, 2012. Biblioteca Virtual Miguel de Cervantes. Web. 1 Sept. 2016.

Gimeno Menéndez, Francisco y María Victoria Gimeno Menéndez. El desplazamiento lingüistico del español por el inglés. Madrid: Cátedra, 2003. Impreso.

Gumperz, John J. Discourse Strategies, Cambridge: Cambridge University Press, 1982. Print.

Gutiérrez Eugenio, Esther. “El uso de otras lenguas en el aula de ELE: resultados de un estudio piloto". Actas del II Encuentro Internacional de ELE del Instituto Cervantes de Bruselas. (2013): 77-89. Centro Virtual Cervantes. Web. 25 Nov. 2016.

Jessner, Ulrike. "Teaching third languages: Findings, trends and challenges". Language Teaching, Vol. 1, Núm. 41 (2008): 15-56. Web. 10 Sept. 2016.

Jiménez Sánchez, Daniel. "Dilo en español mejor. Alternancia códica en el discurso del profesor en una clase de ELE. Memoria de Máster. Universidad de Barcelona." Suplementos MarcoELE, Núm. 14 (2012). Web. 10 Sept. 2016.

Macaro, Ernesto. "Analysing Student Teachers' Codeswitching in Foreign Language Classrooms: Theories and Decision Making". The Modern Language Journal, 4, 85 (2001): 531-548. Print.

Moreno Fernández, Francisco. "Aportes de la sociolingüística a la enseñanza de lenguas". REALE, Núm. 1, (1994): 107-135. Web. 1 Sept. 2016.

-. Adquisición de segundas lenguas: variación y contexto social. Madrid: Arco Libros, 2000. Impreso.

Palikuća, Amila y Valeria Novelliere. "Estudio sobre el uso del inglés en los procesos de eseñanza-aprendizaje en el aula de ELE". Actas del I Encuentro Internacional de profesores de ELE del Instituto Cervantes de Bruselas. 2012. 114-132. Centro Virtual Cervantes. Web. 1 Sept. 2016.

Poplack, Shana. "Sometimes l'll start a sentence in Spanish y termino en español: Toward a typology of code-switching". Linguistics, 18 (1980): 581-618. Print. 